\title{
因HAD
}

ISSN-L: 2530-5115

DOI: http://doi.org/10.22585/hospdomic.v4i3.108

\section{El modelo SciELO en España: un proyecto pionero de acceso abierto}

\section{The SciELO model in Spain: a pioneering open access project}

Cristina Bojo Canales', Cristina A. Fraga Medín', Elena Primo-Peña'

1. Instituto de salud Carlos III, Biblioteca Nacional de Ciencias de la Salud, Madrid, España.

Correspondencia/Correspondence

Cristina Bojo Canales.

Instituto de Salud Carlos III, Biblioteca Nacional de Ciencias de la Salud. Avenida Monforte de

Lemos 5, 28029, Madrid, España.

cbojo@isciii.es

http://orcid.org/0000-0002-0330-171X

Recibido/Received

05.05.2020

Aceptado/Accepted

12.06.2020
Conflicto de Intereses/Competing interest Las autoras declaran la no existencia de conflicto de interés

Financiación/Fundings

No existe

CÓMO CITAR ESTE TRABAJO | HOW TO CITE THIS PAPER

Bojo-Canales C, Fraga-Medín CA, Primo-Peña E. El modelo SciELO en España: un proyecto pionero de acceso abierto. Hosp Domic. 2020;4(3): 153-9. 


\section{RESUMEN}

El proyecto SciELO se constituye como una iniciativa pionera del movimiento de acceso abierto para la publicación de revistas científicas editadas en países en desarrollo y de habla no inglesa.

El artículo comienza revisando los orígenes del proyecto SciELO en Brasil en 1998 y su desarrollo como red cooperativa extendida a 14 países iberoamericanos y Sudáfrica. A continuación, se centra en la iniciativa española, SciELO España, que comienza a funcionar en 2001 con 4 revistas y que en la actualidad incluye 60 revistas y más de 40.000 artículos. Asimismo, se destacan los hitos conseguidos a nivel científico y de respaldo de la comunidad de editores científicos, como lo demuestran las cifras de visitas y descarga de contenidos que recibe, así como la incorporación de sus revistas en índices internacionales de prestigio como Web of Science y Scopus.

Palabras clave: SciELO; Acceso Abierto; Revistas Científicas.

\section{ABSTRACT}

The SciELO project is a pioneering initiative of the Open Access movement for the publication of scientific journals in developing and non-English-speaking countries.

The article begins by reviewing the origins of the SciELO project in Brazil in 1998 and its development as an extended cooperative network to 14 Ibero-American countries and South Africa. It then focuses on the Spanish initiative, SciELO Spain, which begins operating in 2001 with 4 journals and currently includes 60 journals and more than 40,000 articles. It also highlights the milestones achieved at the scientific level and support of the publishing community, as evidenced by the number of visits and content downloads hat it receives, as well as the incorporation of its journals in prestigious international indexes such as Web of Science and Scopus.

Keywords: SciELO; Open Access; Scientific Journals. 


\section{INTRODUCCIÓN}

SciELO surgió en Brasil hace más de dos décadas antes de la publicación de las tres declaraciones que sustentan ideológicamente el movimiento de acceso abierto y que son conocidas como "las tres BBB" (Budapest, Berlín y Bethesda) (1-3) y cuando los índices internacionales limitaban su cobertura a las llamadas revistas de corriente principal ignorando un universo de revistas editadas en países en desarrollo y de habla no inglesa (4-5). El objetivo de SciELO era contribuir al avance de la investigación científica generada en los países Ibero-Latinoamericanos, mejorando la calidad de sus revistas y aumentando su visibilidad, accesibilidad, uso e impacto (4).

Para ello, se desarrolló una metodología de publicación electrónica de revistas científicas (inicialmente se trataba de revistas biomédicas brasileñas) que aprovechaba la red de Internet y las nuevas tecnologías disponibles en aquellos momentos para transformar las revistas impresas en electrónicas y ofrecer los textos completos de los artículos de forma completamente gratuita, adelantándose así en unos años a la modalidad de publicación conocida como Golden Road (6). Además, SciELO proporcionaba una base de datos de indicadores bibliométricos sobre el impacto de las publicaciones basado en la citación que permitía la elaboración de estudios bibliométricos sobre la literatura generada en estos países y que servirían de complemento a los ofrecidos por las bases de datos del antiguo Institute for Scientific Information (ISI).

Asimismo, el modelo SciELO tiene, un componente organizativo que gira alrededor de la evaluación de la calidad de las revistas que se incluyen en cada uno de los portales. Existen unos criterios de calidad generales adaptados a cada portal nacional. De forma general se refieren principalmente al contenido científico de las publicaciones, a la no endogamia, a la utilización del sistema de revisión por pares y al cumplimiento de estándares internacionales de publicación. Estos criterios se complementan con la existencia de un comité consultivo que es el responsable de su vigilancia y aplicación para la entrada y mantenimiento de las publicaciones en la colección.

El proyecto piloto de SciELO se inicia en Brasil en el año 1998 con 10 revistas, liderado por la Fundação de Amparo à Pesquisa do Estado de São Paulo (FAPESP), una de las principales agencias de investigación del país y el Centro Latinoamericano y del Caribe de Información en Ciencias de la Salud (BIREME), organismo dependiente de la OPS/OMS.

Su rápido éxito hizo que el modelo SciELO fuese muy pronto adoptado por Chile y poco a poco se extendiera a otros países de América Latina, conformando una red de colecciones SciELO que operan de manera cooperativa compartiendo una misma metodología para la preparación, almacenamiento, difusión y evaluación de la literatura científica. Las colecciones son gestionadas de manera descentralizada y mantenidas por instituciones nacionales relacionadas con el fomento y apoyo a la investigación científica y a la comunicación de resultados.

Hoy en día, SciELO (www.scielo.org) funciona como una gran red de información científica que cubre 14 países iberoamericanos más Sudáfrica y la colección temática multinacional SciELO Salud Pública. En conjunto, la red indiza más de 1.440 revistas de todas las disciplinas, 452 del área de las ciencias de la salud y da acceso a más de 700.000 artículos. Con un incremento medio de más de 40.000 artículos por año, la Red SciELO recibe una media de más de 1,5 millones de descargas diarias (4-5).

SciELO fue pionero en la adopción del Acceso Abierto y se ha convertido en uno de los principales proyectos de acceso abierto a la ciencia a nivel mundial y sin duda el más importante entre los países en desarrollo y emergentes. La formalización del Acceso Abierto al nivel de las revistas y de los artículos se realiza mediante la adopción de atribuciones de acceso de acuerdo con las licencias Creative Commons (CC) que incentivan la reutilización y distribución de los artículos permitiendo a la vez que el autor mantenga los derechos que considere convenientes. 
A través de los comités consultivos, constituidos por expertos en distintas áreas temáticas y aspectos de la publicación científica, SciELO cuenta con un sistema de control de garantía de la calidad de su contenido, y los procesos evaluativos sirven para orientar los esfuerzos realizados por los editores para fortalecer, perfeccionar y mejorar la calidad y el impacto de las revistas evaluadas y recogidas en cada colección.

Uno de los resultados más importantes de la red SciELO es que los metadatos de sus artículos son recolectados semanalmente en Google Scholar (http://scholar.google.es), Crossref (http:// crossref.org), DOAJ (http://doaj.org) y Open Aire (http://openaire.eu). SciELO España es también proveedor de contenidos de agregadores como Hispana https://hispana.mcu.es/es/inicio/inicio.do) o Recolecta (https://recolecta.fecyt.es/).

Packer et al. (1-2) señalaban los principales beneficios que supone para las revistas formar parte de SCIELO:

- Los textos completos de las revistas se publican en una web interoperable que incrementa en buena medida las posibilidades de localización, recuperación y consulta de los trabajos publicados, puesto que genera metadatos que permiten su indización y recopilación por parte de los motores de búsqueda.

- A través de SciELO todas las revistas quedan indexadas en Google Académico y Crossref.

- Las revistas incluidas en Web of Science, Scopus y MEDLINE pueden interoperar con estas bases de datos mediante el envío de metadatos y enlaces que aumentará su uso e impacto.

- SciELO proporciona toda una batería de indicadores bibliométricos que permite evaluar a las revistas en cuanto a citas, así como en la descarga de artículos. Las métricas basadas en citas, como ocurre en todos los índices de este tipo, quedan limitadas al universo nacional y regional de revistas SciELO.

- Todas las colecciones SciELO certificadas se integran en el SciELO Citation Index, una base de datos que opera dentro de la Web of Science (WOS) en conjunto con otras bases de datos.

- El hecho de que las revistas incluidas en SciELO formen parte de un proyecto colaborativo internacional, respaldado por la Organización Panamericana de la Salud, que ha ido progresando desde sus inicios en 1997 y ahora es un proyecto consolidado de reconocido prestigio, redunda en un gran incremento de su visibilidad y difusión internacional.

- Los diferentes equipos SciELO prestan asistencia para mejorar la gestión editorial de las revistas incluidas en el portal.

\section{SciELO España}

La Biblioteca Nacional de Ciencias de la Salud del Instituto de Salud Carlos III puso en marcha el sitio SciELO España (http://scielo.isciii.es ) en el año 2001 con una colección inicial de cuatro títulos (7). En el año 2003, coincidiendo con el VI Congreso Regional de Información en Ciencias de la Salud, se anunció oficialmente la integración de SciELO España en la Red SciELO y desde entonces está accesible a través del sitio www.scielo.org.

Desde el año 2008, SciELO España cuenta con un comité consultivo formado por representantes de la comunidad relacionada directamente con la comunicación científica a nivel nacional e incluye a editores de revistas científicas, expertos en evaluación de la ciencia y de la comunicación científica, en Open Access y políticas de acceso abierto, documentalistas e investigadores del área de las ciencias de la salud. Hasta el momento se han celebrado 8 reuniones y dos jornadas con editores de revistas en SciELO. 
SciELO nació dentro del ámbito de las revistas de ciencias de la salud, pero, poco a poco, todas las colecciones nacionales se han transformado en multidisciplinares. SciELO España mantiene su rasgo monográfico y recoge revistas del área de medicina, enfermería, farmacia, psicología y odontología (gráfico 1).

\section{Gráfico 1: áreas temáticas de las revistas SciELO España}

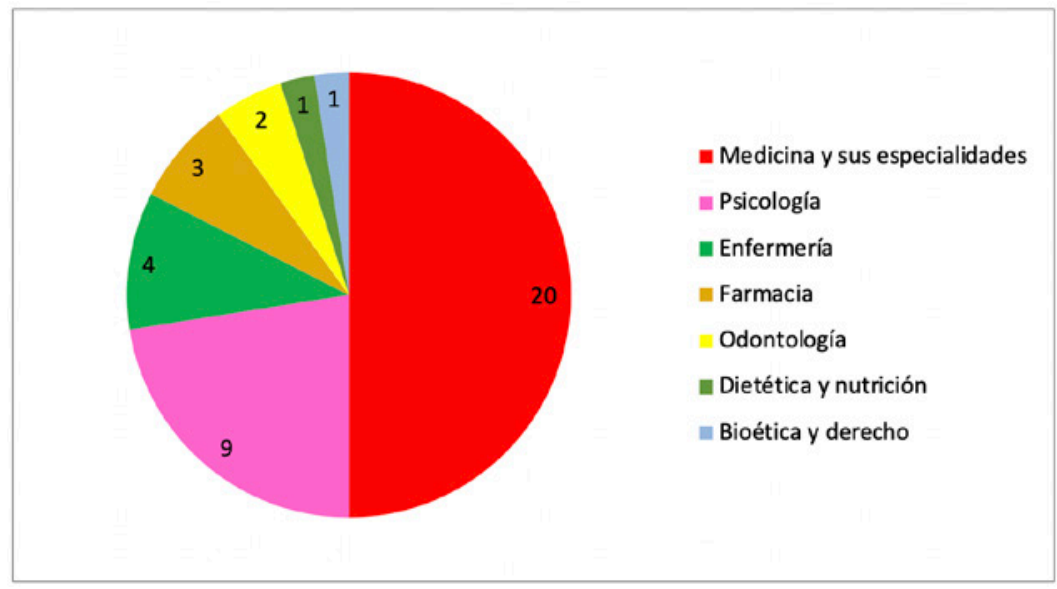

SciELO España es un proyecto dinámico que a lo largo de estos años ha ido incrementando su colección, de tal modo que en estos momentos son 60 las revistas presentes (40 vigentes) lo que suponen casi 3000 fascículos, unos 40.000 artículos y más de 800.000 referencias (Gráfico 2).

Gráfico 2: crecimiento de artículos de SciELO España durante el periodo 2001-2020

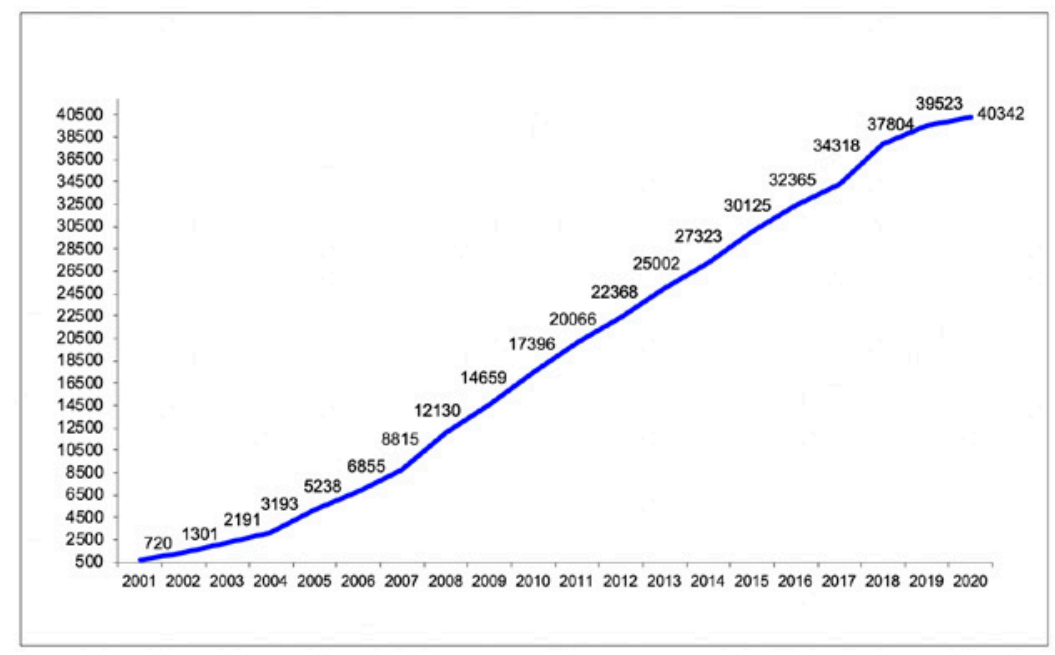


El impacto que la colección SciELO España tiene entre la comunidad científica y académica queda evidenciado en el elevado número de visitas y descargas de artículos. Durante el pasado año 2019 recibió más de 36,5 millones de visitas, con una media de más de 3 millones al mes. Asimismo, estas visitas supusieron la descarga de más de 43 millones de páginas.

Respecto al tipo de editorial, son mayoría las revistas editadas por sociedades científicas, asociaciones y colegios profesionales (gráfico 3), aunque también están presentes revistas editadas por otro tipo de editores, entre ellos, editoriales universitarias y de departamentos de salud.

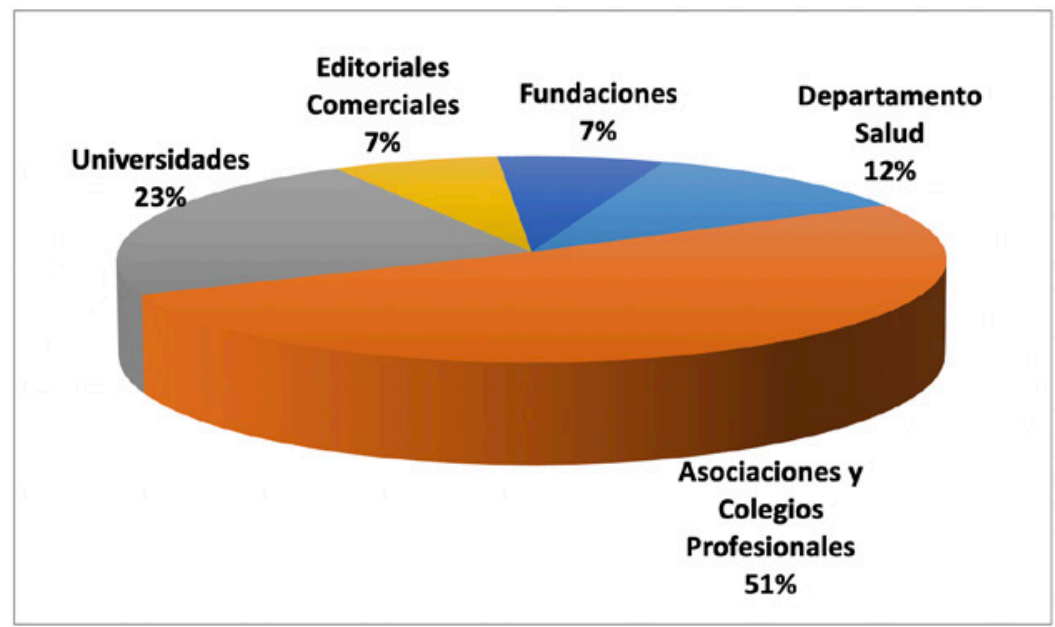

El idioma en el que están publicadas las revistas se puede observar en el gráfico 4

Gráfico 4: Idiomas de publicación de las revistas SciELO España

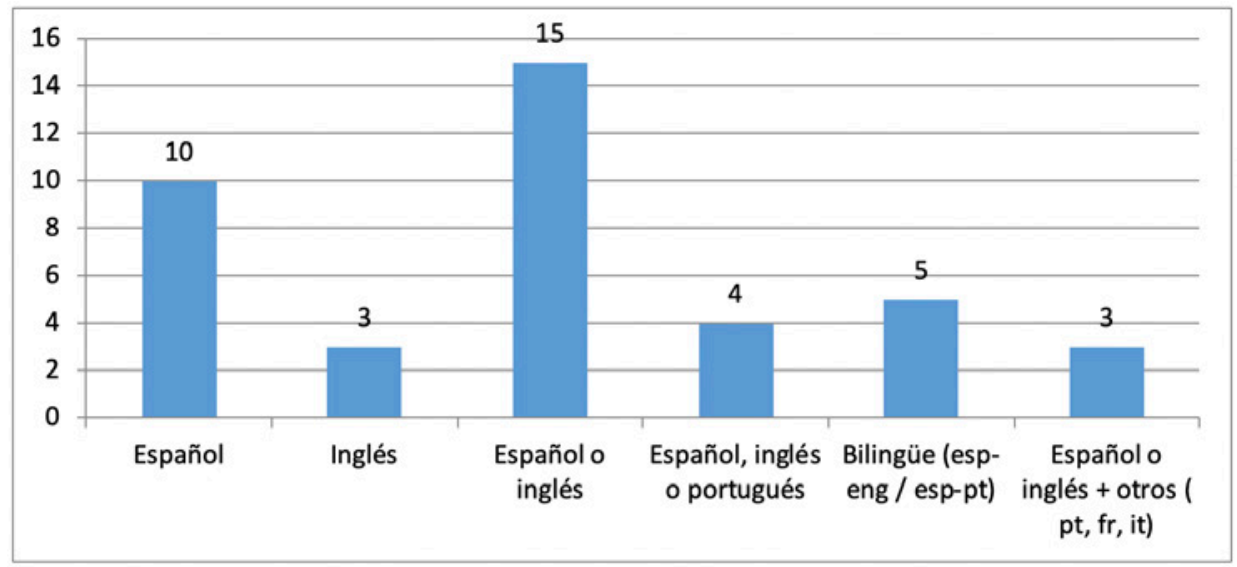


Tan solo el 12\% $(n=5)$ de las revistas de SciELO España cobra tasas de publicación (APC por sus siglas en inglés) con un precio que varía entre los 100 y los 450 euros por artículo. El resto son financiadas por las instituciones editoras (Sociedades, Universidades, ...)

Hasta el momento, el 63\% de las revistas de la colección española han adoptado una Licencia CC, siendo la de tipo BY-NC-ND (Reconocimiento-NoComercial-SinObraDerivada) la más utilizada. Esta licencia permite que otros puedan descargar las obras y compartirlas con otras personas, siempre que se reconozca su autoría, sin que pueda modificarse ni utilizarse comercialmente.

Tras más de 20 años de funcionamiento puede decirse que SciELO fue un proyecto pionero y decisivo en el movimiento mundial a favor del Acceso Abierto. Hoy es una iniciativa consolidada y mundialmente reconocida en la comunidad académica y de investigación.

\section{BIBLIOGRAFÍA}

1. Iniciativa de Budapest para el acceso abierto [monografía en Internet]. Nueva York, EE.UU.: Open Society Institute; 2001 [citado 1 mayo 2020]. Disponible en: https://www.budapestopenaccessinitiative.org/esp/index

2. Berlin Declaration on Open Access to Knowledge in the Sciences and Humanities [monografía en Internet]. Múnich, Alemania: Sociedad Max Planck; 2003 [citado 1 mayo 2020]. Disponible en: https://openaccess.mpg.de/Berlin-Declaration

3. Bethesda Statement on Open Access Publishing [monografía en Internet]. Bethesda, EE.UU.: National Library of Medicine; 2003 [citado 1 mayo 2020]. Disponible en: https://bit.ly/3h6GoHf

4. Packer AL, Co N, Luccisano A, Ramalho A, Spinak, E. SciELO: 15 Años de Acceso Abierto Un estudio analítico sobre Acceso Abierto y comunicación científica. Paris, Francia: UNESCO; 2014. DOI: $10.7476 / 9789233012370$

5. Packer AL, Meneghini R. Contribución de SciELO a la Globalización de la Ciencia [monografía en Internet]. Sao Paulo, Brasil: Scientific Electronic Library Online; 2015 [Fecha de consulta 17/05/2020]. Disponible en: https://bit.ly/2ZIF6St

6. Packer AL. The SciELO Open Access: A Gold Way from the South. Can J High Educ. 2009;39(3):111-26.

7. Bojo Canales C. La red SciELO (Scientific Electronic Library Online): perspectiva tras 20 años de funcionamiento. Hosp Domic. 2017;1(4):211-20. DOI: 10.22585/hospdomic.v1i4.31 